\title{
Immune responses to the I05AD7 human anti-idiotypic vaccine after intensive chemotherapy, for osteosarcoma
}

\author{
K Pritchard-Jones ${ }^{1,10}$, I Spendlove ${ }^{2,10}$, C Wilton ${ }^{2}$, J Whelan ${ }^{3}$, S Weeden ${ }^{4}$, I Lewis ${ }^{5}$, J Hale ${ }^{6}$, C Douglas ${ }^{7}$, \\ C Pagonis ${ }^{8}$, B Campbell ${ }^{8}$, P Alvarez ${ }^{8}$, G Halbert ${ }^{9}$ and LG Durrant ${ }^{*, 2,10}$
}

'Royal Marsden Hospital and Institute of Cancer Research, Downs Road, Sutton, Surrey SM2 5PT, UK; ${ }^{2}$ The Department of Clinical Oncology, The University of Nottingham, City Hospital, Nottingham NG5 IPB, UK; ${ }^{3}$ Middlesex Hospital, Nassau Street, London WIT 3AA, UK; ${ }^{4}$ Cancer Division, MRC Clinical Trials Unit, 222 Euston Road, London NWI 2DA, UK; ${ }^{5}$ St James's University Hospital, Beckett Street, Leeds, West Yorkshire LS9 7TF, UK;

${ }^{6}$ Newcastle General Hospital, Westgate Road, Newcastle-upon-Tyne NE4 6BE, UK; ${ }^{7}$ UKCCSG, University of Leicester, 9 Princess Road West, Leicester LEI 6TH, UK; ${ }^{8}$ Cancer Research UK Drug Development Office, 61 Lincoln's Inn Fields, London WC2A 3PX, UK; ${ }^{9}$ Cancer Research UK Formulation Unit, Department of Pharmaceutical Sciences, Royal College Building, University of Strathclyde, 204 George Street, Glasgow GI IXW, UK

105AD7 is a human monoclonal antibody that mimics the complement regulatory protein, CD55, overexpressed by many solid tumours including osteosarcoma. This study was designed to assess the toxicity and efficacy of this vaccine in a young age group of patients within I-6 months of myleosuppressive chemotherapy. Out of 28, 20 (7I\%, 95\% Cl 5I -87\%) patients showed a significant T-cell proliferation response in vitro to the I05AD7 protein but not to human IgG. Furthermore, I 3 out of 22 (59\%, 95\% Cl 36-79\%) patients showed antigen-specific $\gamma$ IFN secretion (range $20-370 \mathrm{U} / \mathrm{ml}$ ). Nine out of $28(32 \%, 95 \% \mathrm{Cl}$ I6-52\%) patients made weak antibody responses to CD55. This study showed that I05AD7 was well tolerated in younger patients with osteosarcoma. In addition, two patients with possible clinical responses were given compassionate permission to continue immunisation quarterly for 2 years. They both remain alive and disease free 5.8 and 6.5 years from original diagnosis of osteosarcoma and showed no adverse effects of repeated immunisation. In conclusion, the majority of patients showed measurable $T$ helper responses when vaccination was commenced within a 6-month window of intensive chemotherapy with no clinically significant toxicity. Future clinical trials incorporating immune stimulation strategies should include early introduction of vaccines during the highest risk period for relapse. British Journal of Cancer (2005) 92, I358- 1365. doi:I0.1038/sj.bjc.6602500 www.bjcancer.com

Published online 29 March 2005

(c) 2005 Cancer Research UK

Keywords: cancer vaccines; osteosarcoma; clinical trial; immune responses; anti-idiotypic antibodies

Osteosarcoma is a high-grade bone tumour that affects mainly adolescents and young adults. Two-thirds of patients diagnosed before the age of 30 years have a localised primary tumour in a limb bone. Of these, about $50-60 \%$ can expect to be long-term disease-free survivors with modern therapies that include intensive multiagent chemotherapy and surgical resection (Souhami et al, 1997; Fuchs et al, 1998). The remaining one-third of patients with osteosarcoma have a poorer prognosis and present with either a primary in the axial skeleton or metastatic disease. Even among localised disease, most relapses occur within 2 years of initial diagnosis, and a third of patients relapse or die within 6 months of the scheduled end of multiagent chemotherapy. Comparisons of the several national and international clinical trials of manipulation of chemotherapy type and dose intensity suggest a limited role for conventional chemotherapy in further improvements in disease control. Hence, there is a need to search for novel approaches.

\footnotetext{
*Correspondence: Dr LG Durrant;

E-mail: lindy.durrant@nottingham.ac.uk

${ }^{10}$ These authors contributed equally to this work

Received 25 November 2004; revised 3 February 2005; accepted 7 February 2005; published online 29 March 2005
}

A possible therapeutic role for immunomodulation in osteosarcoma was suggested by the naturally occurring dog model of this disease, in which injections of the macrophage activator, liposomal encapsulated muramyl tripeptide phosphatidyl ethanolamine (MTP-PE) induced regression. The role of MTP-PE as an immune stimulatory adjuvant therapy was tested in a recent trial conducted by the Children's Oncology Group in North America. This $2 \times 2$ factorial trial randomised patients to receive control chemotherapy (methotrexate, doxorubicin and cisplatin) with or without the addition of ifosfamide and with or without MTP-PE. Preliminary results of this trial have indicated that although neither treatment offers an event-free survival benefit individually, there is a synergistic effect when ifosfamide and MTP-PE are administered together (Meyers et al, 2001) Although suggestive, this study does not provide conclusive evidence of the benefit from this form of immunomodulation.

In order to impact on the early relapses, any immunotherapy approach would have to be commenced relatively early in the course of treatment. Since the chemotherapy given for osteosarcoma is very myelosuppressive, we wished to test whether patients could mount an immune response to a relevant tumour antigen if a vaccination strategy was commenced within 1-6 months of completion of chemotherapy. We chose to use an anti-idiotypic 
monoclonal antibody, 105AD7, whose safety had been demonstrated in two previous trials in patients with colorectal cancer.

105AD7 is a human anti-idiotypic monoclonal antibody that was cloned from a cancer patient who had received the radiolabelled mouse monoclonal 791T/36 for diagnostic imaging (Austin et al, 1989). 791T/36 was subsequently shown to recognise CD55, a complement regulatory protein overexpressed by tumours to protect them from complement attack. 105AD7 was shown to have both amino acid and structural homology with CD55 and could therefore be used as a vaccine to induce antitumour immune responses (Spendlove et al, 2000).

In a phase I clinical trial in metastatic colorectal cancer patients, 105AD7 induced antitumour inflammatory responses with no associated toxicity (Denton et al, 1994). However, in a subsequent double-blind randomised phase II trial there was no survival benefit in the setting of measurable disease (Maxwell-Armstrong et al, 2001). This may have been due to the immunosuppressive effects of large tumour burden as a neoadjuvant trial showed promising results indicating a valuable immune response (Durrant et al, 2000a, b; Schwann et al, 2000). As 791T/36 was originally raised against a human osteogenic tumour cell line and was used successfully to image osteosarcomas in vivo (Farrands et al, 1983), we proposed that $105 \mathrm{AD} 7$ may be a suitable vaccine for treatment of this disease. Previous experience with colorectal cancer suggested that 105AD7 would be most effective in treatment of patients with minimal residual disease.

This study had two main aims: to assess the toxicity of vaccinating osteosarcoma patients with 105AD7 and to determine the ability of these patients to raise an immune response to this vaccine.

\section{MATERIALS AND METHODS}

\section{Patients}

Patients were recruited from four centres in England (The Middlesex Hospital, London; Royal Marsden Hospital, Sutton, Royal Victoria Infirmary, Newcastle and St James Hospital, Leeds). Eligible patients had a diagnosis of high-grade osteosarcoma, were aged $<30$ years at time of study entry and had a lymphocyte count of $>0.5 \times 10^{9} \mathrm{ml}^{-1}$, and biochemical indices of renal and liver function within three times the age-related upper limit of normal.

Patients were recruited if they were deemed at high risk of treatment failure because they had presented with metastatic disease, had experienced at least one relapse or if they presented with a localised limb primary tumour and who were not participating in another clinical trial. Patients who generally have a better outlook were included in this phase I/II study due to the good safety profile of the vaccine in previous studies in adult colorectal cancer patients. All patients had to have completed current best standard therapy for their situation and to have received their last dose of chemotherapy within the previous 1-6 months. All patients had a Lansky performance score of $>80$ or a Karnofsky performance score of $>60 \%$, according to age. Exclusion criteria were any autoimmune disease, lymphocyte count $<0.5 \times 10^{9} 1^{-1}$, platelet count $50 \times 10^{9} 1^{-1}$, concomitant anticancer treatment or other vaccinations within the previous 3 weeks. All patients (and their parents when the patient was aged less than 16 years) gave fully informed, written consent to participate in the study.

Patients were required to have baseline imaging studies, appropriate to their clinical situation and follow-up, immediately prior to study entry, to confirm either clinical remission or presence of disease. Patients with measurable disease were required to have the appropriate imaging modality repeated at week 9, to look for response, and again at week 14, to assess durability of response.

\section{Vaccination schedule and venous blood sample handling}

The vaccination schedule and blood sampling are shown in Figure 1. For isolation of lymphocytes, a $20 \mathrm{ml}$ baseline venous blood sample was taken in preservative-free heparin during the week prior to commencement of vaccination and again at week 0 , $3,6,9,12$ and 15. Full blood count, urea, electrolytes, creatinine and liver function tests were performed at weeks $0,3,6$ and 12 at the clinical trial centre for that patient. Patients received an intradermal (i.d.) injection of $10 \mu \mathrm{g}$ of $105 \mathrm{AD} 7$ and an intramuscular (i.m.) dose of $100 \mu \mathrm{g}$ of 105AD7 adsorbed on alyhdrogel 85 at weeks $0,3,6$ and 12, immediately following the blood sampling. The venous blood samples were sent by overnight courier to the immune analysis laboratory where lymphocytes were separated on Lymphoprep (Flow Laboratories, Irvine, Scotland) and stored at $-180^{\circ} \mathrm{C}$. Plasma was also stored at $-80^{\circ} \mathrm{C}$.

This trial was carried out under the auspices of the Cancer Research UK Drug Development Office with local ethical approval in all centres. The study was carried out in accordance with the Declaration of Helsinki 1996.

\section{Antibody}

Clinical grade 105AD7 human antibody was produced as described previously using the guidelines of the Cancer Research Campaign, United Kingdom (Denton et al, 1994). The trial supplies were prepared under cGMP conditions. Antibody for immunisation was prepared adsorbed on alhydrogel 85 (Superfos Biosector now Brenntag Biosector A/S, Elsenbakken, Denmark) at $100 \mu \mathrm{g} \mathrm{ml}^{-1}$ in sterile, pyrogen-free phosphate-buffered saline in a $1 \mathrm{ml}$ type 1 glass ampoule containing $1 \mathrm{ml}$. The weight ratio of 105AD7 to alhydrogel 85 was $1: 10$. The skin test dose was prepared at $100 \mu \mathrm{g} \mathrm{ml}^{-1}$ in sterile, pyrogen-free phosphate-buffered saline in a 1 -ml type 1 glass ampoule containing $0.125 \mathrm{ml}$. Stability studies have shown that the antibody can be stored at $4{ }^{\circ} \mathrm{C}$ for a minimum of 5 years with no loss in binding activity.

\section{T-cell analysis}

A complete set of cryopreserved lymphocytes for each patient was defrosted for simultaneous analysis. A sample of peripheral blood mononuclear cells (PBMCs) was removed, irradiated and used as antigen-presenting cells. The remaining PBMCs were stimulated with these antigen-presenting cells in the presence of 105AD7 $\left(10 \mu \mathrm{g} \mathrm{ml}^{-1}\right)$ or control human IgG antibody $\left(10 \mu \mathrm{g} \mathrm{ml}^{-1}\right)$ in RPMI (Flow laboratories) containing $10 \%$ allogeneic human serum at $10^{6} \mathrm{cell} \mathrm{ml}^{-1}$ in 24 -well plates. All cultures were incubated in a humidified incubator of $5 \% \mathrm{CO}_{2}$ and $95 \%$ air at $37^{\circ} \mathrm{C}$. Proliferation of lymphocytes was quantified after 5 days by resuspending the cells and removing four $100 \mu \mathrm{l}$ aliquots and incubating these cells in fresh 96-well plates overnight in the presence of $3[\mathrm{H}]$ thymidine. All experiments were in quadruplicate and there was always an s.d. of less than $10 \%$. Each time course was repeated twice and if there was any discordance it was repeated a third time. The response to human IgG was not significantly different from the medium control by a one-way analysis of variance. For clarity, results on figures and tables are presented as a stimulation index $(\mathrm{SI})=$ incorporated counts per minute $(\mathrm{cpm})$ in the presence of $105 \mathrm{AD} 7 / \mathrm{cpm}$ in the presence of Human IgG. One-way analysis of variance showed that all samples that responded with a two-fold increase in response to $105 \mathrm{AD} 7$ as compared to human IgG were significant.

\section{$\gamma$ IFN Secretion}

$\gamma$ IFN secretion was analysed in tissue culture supernatant of lymphocytes that had been stimulated with either 105AD7 
A

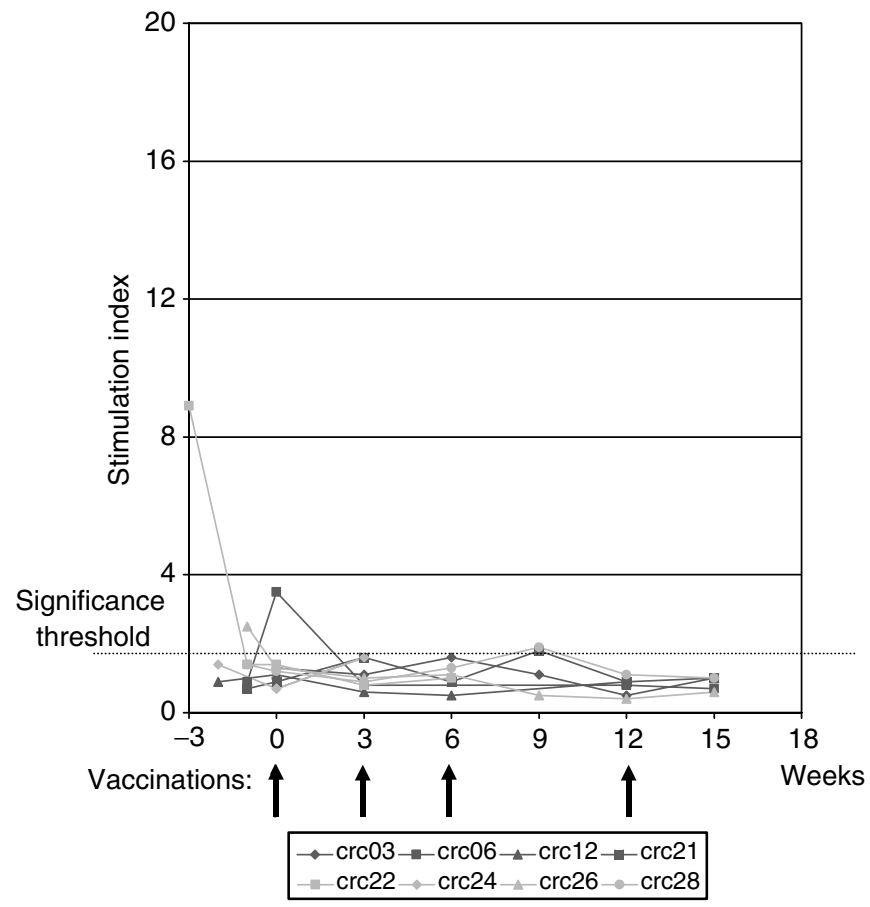

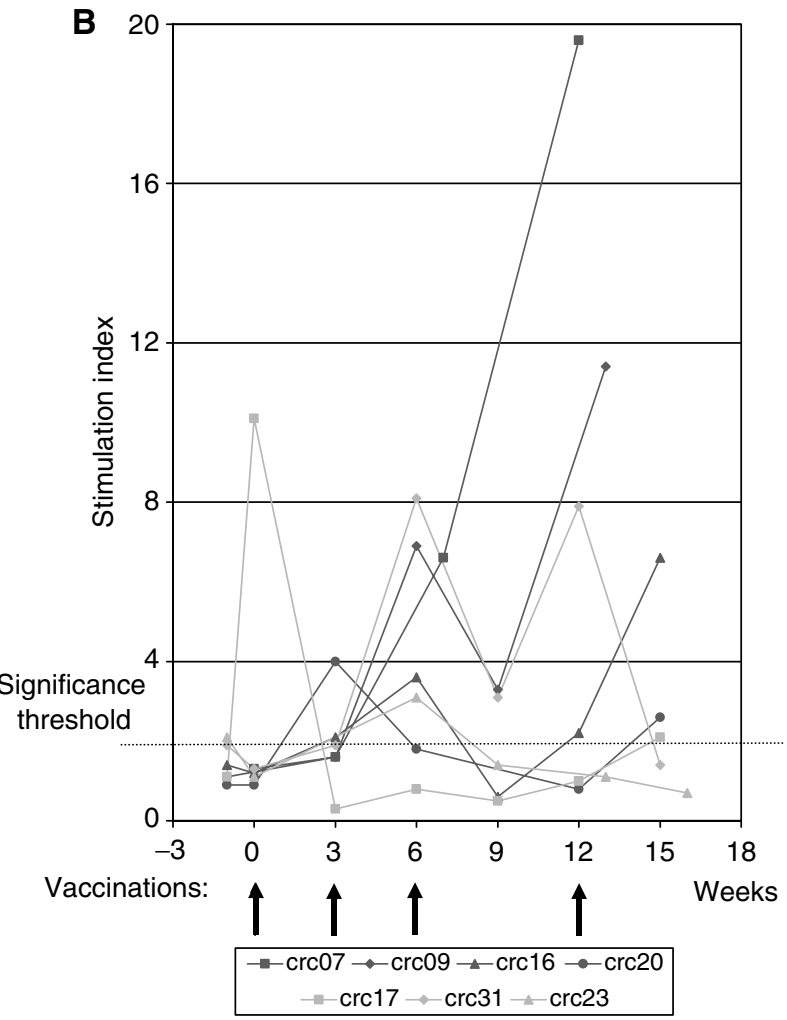

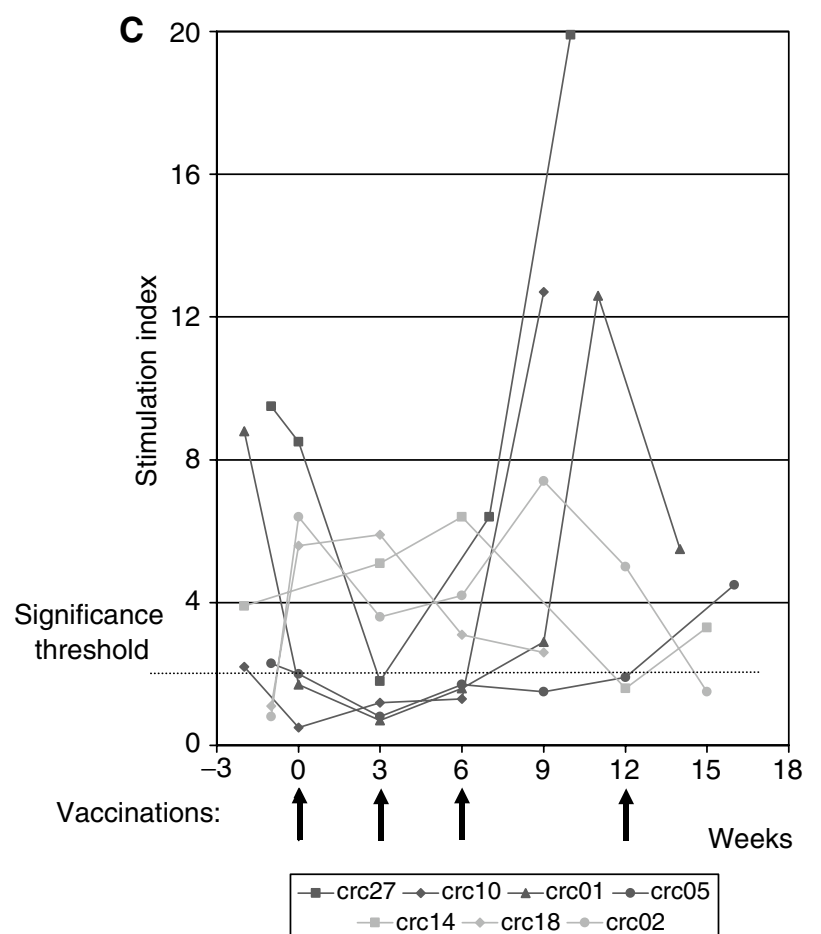

Figure I (A) Patients showing no proliferative response to I05AD7 or human IgG control. (B) Patients showing proliferative response to I05AD7 but not human $\operatorname{lgG}$ control (no prevaccination response). (C) Patients showing proliferative response to I05AD7 but not human lgG control (with prevaccination response). T-cell proliferation was assessed by ${ }^{3} \mathrm{H}$-labelled thymidine incorporation following 5 -day stimulation with either $105 \mathrm{AD} 7$ or control human lgG:

$$
\mathrm{SI}=\frac{\mathrm{cpm} 105 \mathrm{AD} 7}{\mathrm{cpm} \text { hIgG }}
$$

An SI of greater than 2 is considered significant. Arrows denote injection with I05AD7 by intrademal ( $10 \mu \mathrm{g})$ and intramuscular (I00 $\mu \mathrm{g}$ on alum) injection. 
$\left(10 \mu \mathrm{g} \mathrm{ml}^{-1}\right)$ or human IgG $\left(10 \mu \mathrm{g} \mathrm{ml}^{-1}\right)$ for 5 days in vitro $\gamma \mathrm{IFN}$ was detected by ELISA (R\&D, Abingdon, Oxon, UK).

\section{Antibody response}

CD55 and CEA were purified from 791Tcells and colorectal tumours using previously described methods (Price et al, 1987; Spendlove et al, 1999) and used to coat microtitre plates by overnight incubation at room temperature. Plates were blocked with phosphate buffer and saline (PBS) containing 1\% bovine serum albumin (BSA). Plasma samples were defrosted, diluted in PBS containing $0.1 \%$ BSA and incubated with the antigens for $1 \mathrm{~h}$ at $4{ }^{\circ} \mathrm{C}$. Bound antibodies were detected by rabbit anti-human horseradish peroxidase (Dako, Denmark) and TMB substrate ( $R$ and $D$ ). Absorbance was read at $402 \mathrm{~mm}$ on a plate reader.

\section{HLA typing}

Patients MHC class II phenotyping was measured by standard molecular techniques.

\section{Statistical methodology}

Due to the small number of patients available to the study and the fact that this vaccine had not previously been tested in osteosarcoma, the sample size was determined using the single stage procedure proposed by Fleming (1982). This method makes use of the response probabilities of interest, and allows statistically meaningful designs to be constructed with relatively small patient numbers. Sample sizes for Fleming's single stage procedure are tabulated by A'Hern (2001). The lowest response probability of interest is set as 0.50 and the highest is set as 0.75 . To reliably evaluate the immunological response rate for the new vaccine ( significance level $=5 \%$, power $=80 \%$ ), 23 patients would have to be entered into the study.

Statistical analysis was carried out using SPSS Version 1 for Windows. Exact 95\% confidence intervals (CI) for response rates were obtained from Geigy Scientific Tables (Altman et al, 2000).

\section{RESULTS}

This trial had two main aims. The phase I component was to assess the toxicity profile of this agent in children and young adults, since the previous excellent safety profile had been documented only in older adults with colorectal cancer. The phase II component addressed the question of whether 105AD7 was capable of stimulating a specific immune response in this relatively heavily pretreated group of patients within 1-6 months of their last dose of chemotherapy. The evaluation of any clinical responses where possible was as a secondary end point

In total, 31 patients were recruited to the study from August 1998 to January 2001. One withdrew prior to receiving any vaccine as a baseline CT scan showed recurrent disease. Two further patients withdrew after one or two doses of vaccine due to early progressive disease. The remaining 28 patients received three (5) or four (23) doses of vaccine. In total, 30 patients were evaluated for toxicity, having received at least one dose of vaccine, and 28 had received at least three doses of vaccine to give sufficient time points of blood sampling for analysis of immune response.

\section{Immune responses}

The immune response of patients to the anti-idiotypic vaccine was assayed by in vitro $\mathrm{T}$-cell proliferation responses following exposure to $105 \mathrm{AD} 7$ and to control human IgG. In a subset of patients, stimulation of secretion of $\gamma$ IFN following the same stimulator was also measured. Overall 20 out of 28 (71\%, 95\% CI $51-87 \%$ ) patients showed a proliferative response to $105 \mathrm{AD} 7$ but not to human Ig. Patients' proliferation responses are summarised in Table 1. Patients PBMC proliferative responses could be divided into three groups: Group I (eight patients) failed to show any significant proliferation response to 105AD7 postvaccination (Figure 1A). Group II (seven patients) showed lymphocyte proliferation in response to the vaccine but had no pre-existing response to 105AD7 (Figure 1B). Group III (13 patients) showed a pre-existing response to $105 \mathrm{AD} 7$, which was subsequently boosted by the vaccine (Figure 1C). Response data for all three groups are summarised in Table 1 and Figure 1.

In contrast to previous studies in chemonaïve colorectal cancer patients who responded to the first vaccination, a median of three doses of vaccine were required to observe a peak PBMC proliferative response in this group of patients with osteaosarcoma. A similar delayed response was observed in the group of patients who had evidence for a pre-existing response to 105AD7.

Table I Immune responses of patients

\begin{tabular}{|c|c|c|c|c|c|}
\hline \multirow[b]{2}{*}{ Patient } & \multirow[b]{2}{*}{$\begin{array}{l}\text { HLA/DR } \\
\text { type }\end{array}$} & \multicolumn{2}{|c|}{$\begin{array}{l}\text { Proliferation } \\
\quad \text { (SI) }\end{array}$} & \multirow[b]{2}{*}{$\begin{array}{c}\text { Number of } \\
\text { injections to } \\
\text { observe response }\end{array}$} & \multirow[b]{2}{*}{$\left(\begin{array}{l}\gamma \mathrm{IFN} \\
\left(\mathbf{U} \mathbf{~ m}^{-1}\right)\end{array}\right.$} \\
\hline & & Pre & Post & & \\
\hline \multicolumn{6}{|c|}{ Group I-Patients who failed to show a proliferative response to 105AD7 or human I } \\
\hline 3 & 1,3 & 1.3 & 1.6 & - & 0 \\
\hline 6 & 13,15 & 0.9 & 1.3 & - & ND \\
\hline 12 & 1,7 & 0.9 & 1.1 & - & ND \\
\hline 21 & 13,15 & 0.9 & 1.8 & - & 0 \\
\hline $22^{b}$ & 11,13 & 2.1 & 1.4 & - & 60 \\
\hline $24^{\mathrm{b}}$ & 4,8 & 1.4 & 1.6 & - & 300 \\
\hline 26 & 1,7 & 1.5 & 1.1 & - & 0 \\
\hline 28 & 7,12 & 1.3 & 1.9 & - & 370 \\
\hline \multicolumn{6}{|c|}{ Group II - Patients who showed a proliferative response to 105 AD7 but not human If } \\
\hline 20 & 7, 11 & 0.9 & 4.0 & I & 370 \\
\hline $9^{\mathrm{a}}$ & 4,11 & I.I & 6.9 & 2 & 45 \\
\hline 23 & 1,7 & I.I & 3.1 & 2 & 0 \\
\hline 31 & ND & 1.3 & 8.0 & 2 & 25 \\
\hline 7 & -17 & 1.3 & 19.6 & 4 & 0 \\
\hline 16 & 4,17 & 1.3 & 6.6 & 4 & 70 \\
\hline 17 & 7,17 & 1.1 & 2.1 & 4 & ND \\
\hline \multicolumn{6}{|c|}{$\begin{array}{l}\text { Group III - Patients who had a pretreatment response to 105AD7 and who also } \\
\text { showed a proliferative response to I05AD7 but not to human Ig }\end{array}$} \\
\hline $18^{\mathrm{b}}$ & 12,7 & 5.6 & 5.9 & । & 130 \\
\hline 14 & 1,4 & 3.9 & 6.4 & 2 & 0 \\
\hline $15^{\mathrm{b}}$ & 3,4 & 2.4 & 6.2 & 2 & ND \\
\hline $1^{\mathrm{a}}$ & 1,7 & 8.8 & 12.6 & 3 & 90 \\
\hline 2 & 3,7 & 6.4 & 7.4 & 3 & 185 \\
\hline 8 & 1,15 & 6.7 & 3.9 & 3 & ND \\
\hline 10 & 1,3 & 2.2 & 12.7 & 3 & ND \\
\hline $27^{\mathrm{b}}$ & 4,13 & 8.5 & 19.9 & 3 & 60 \\
\hline 5 & -15 & 2.3 & 4.5 & 4 & 20 \\
\hline 11 & 4,13 & 9.6 & 4.6 & 4 & 0 \\
\hline 13 & -3 & 4.0 & 2.0 & 4 & 40 \\
\hline 29 & 10,17 & 2.0 & 2.0 & 4 & 0 \\
\hline 4 & & 2.5 & 7.5 & I & 0 \\
\hline \multicolumn{6}{|c|}{ 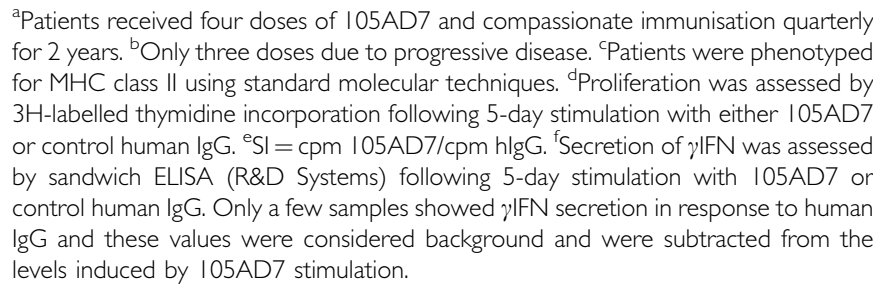 } \\
\hline
\end{tabular}


Previous studies have shown a correlation between response to 105AD7 and an expression of HLA-DR 1, 3, 7 phenotypes. Similar results were observed in this study with 11 out of $15(73 \%)$ of patients expressing these phenotypes showing a proliferation response. However, this study suggests that patients expressing HLA-DR 13, 15 and 17 haplotypes may also respond to 105AD7 vaccine (Table 1 ).

In total, 22 patients were also assayed for secretion of $\gamma$ IFN. A total of 13 patients $(59 \%, 95 \%$ CI $36-79 \%)$ secreted $\gamma$ IFN in response to 105AD7 but not to pooled human immunoglobulin. There was a correlation between the proliferation assay and $\gamma$ IFN secretion in 10 patients. Six patients showed a proliferation response but no significant secretion of $\gamma$ IFN could be observed. Three patients showed $\gamma$ IFN secretion to 105AD7 but failed to have a significant proliferation response. Three patients failed to respond in either assay.

Patients were also screened for their ability to make an antibody response. The antigen 105AD7 mimics is CD55. At a dilution of $1 / 3,11$ out of 28 patients showed a boosted antibody response to CD55 that was greater than the response to CEA (carcinoembryonic antigen; Figure 2). A further six patients had a pre-existing response to $\mathrm{CD} 55$ that was not boosted by vaccination with 105AD7. All but one of these patients was in group III that also showed a pre-existing PBMC proliferative response to 105AD7 (data not shown). Of the nine patients showing an anti-CD55 response only five of these were titratable and two were IgM and three IgG responses.

\section{Toxicity}

Of the 30 patients assessed for toxicity, 15 patients had a minor adverse event (grade 1 or 2 ) that was deemed probably or almost certainly related to administration of 105AD7. These events comprised 33 episodes of local symptoms at the injection site, lasting up to $24 \mathrm{~h}$, and three episodes of intermittent chest pain in one patient and general lethargy in one other.

Two patients experienced exacerbation of tumour-related pain in the week following vaccination, one requiring hospitalisation for

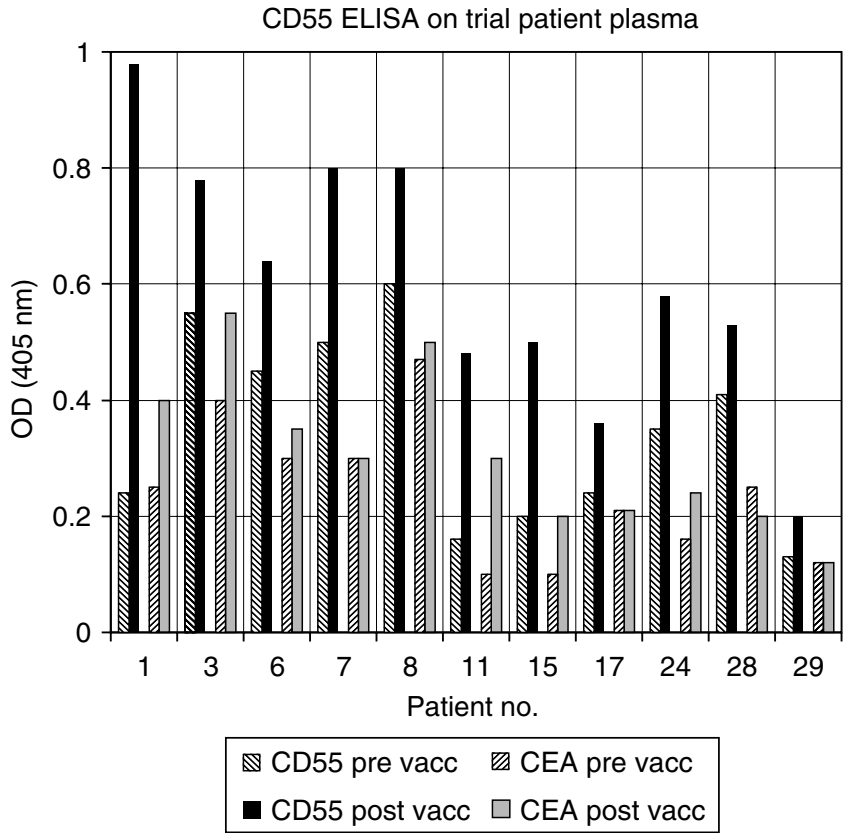

Figure 2 Antibody responses of patients to CD55 and control antigen CEA, pre- and postimmunisation with I05AD7. Antibodies were detected in patients serum by ELISA. intravenous opiates. However, both of these patients had extensive disease at time of study entry, had experienced similar unstable pain prior to study entry and both had subsequent early progressive disease. One patient developed grade 3 lymphopaenia that resolved spontaneously after the second dose of vaccine and did not recur.

Two patients were given prolonged vaccination on compassionate grounds, as there was a possibility that they had had a benefit from the vaccine. They each continued both i.d. and i.m. vaccination on a 2-3 monthly basis until they were 2 years from the initial vaccination. Neither patient experienced any vaccinerelated symptoms. This lack of toxicity is in keeping with previous clinical studies in colorectal cancer patients (Denton et al, 1994; Durrant et al, 2000a; Maxwell-Armstrong et al, 2001).

\section{Disease response}

This study was not designed to test antitumour efficacy. Only five patients entered the study with measurable disease (Table 2). Three patients $(\mathrm{CRC} 15,22,24)$ received only three doses of vaccine due to early disease progression, one patient (CRC7) completed all four doses as sole therapy for an early local recurrence of a pelvic primary tumour but progressed again shortly afterwards. The fifth patient (CRC9) showed evidence of possible response and remains well, as described below.

Table 2 shows the status at study entry and clinical course of all the patients in the trial, grouped according to their in vitro immune response to 105AD7. There were no obvious differences between the three immune response groups in either number of prior relapses, number with measurable disease, clinical risk group or median interval from end of last chemotherapy to study entry (18.5, 21 and 18 weeks for groups I, II and III respectively). In patients who failed to mount an immune response (group I) after 105AD7 vaccination, all those who showed evidence of disease subsequently died of disease. However, in those patients who demonstrated an immune response (Group II and III), there were five patients who remain alive and disease free $(n=3$, at $32,49,61$ months from study entry) or alive with disease $(n=2$ at 34 and 50 months from study entry) although they showed evidence of disease.

Two patients showed unusual clinical courses CRC9 and CRC1. One CRC9 in response group II had presented 12 months prior to study entry with a pelvic primary and lung metastases that showed no response to conventional first line chemotherapy. She was therefore treated with an experimental approach combining highdose chemotherapy and stem cell rescue with systemic intravenous radioactive samarium. Following completion of this, she was found to have residual inoperable lung metastases. As she had shown in vitro responses to 105AD7 (Figure 3A), she was allowed to continue on a compassionate basis with what was believed to be the best potential therapeutic vaccine schedule. CRC9 received a total of a further six doses of vaccine at 3 monthly intervals. Her in vitro immune response fell dramatically after the end of the formal study, suggesting that a memory response was not being established (Figure 3A). However, with resumption of vaccination this was rapidly boosted and reached a peak stimulation index of 32 . Although this then fell to within the unmeasurable range on a single sample taken after completion of the prolonged vaccination, she remains free of signs of progressive disease 4.2 years from study entry and 2.2 years from her last dose of vaccine.

The second patient (CRC1) was in response group III. She had originally been treated for localised distal femoral osteosarcoma and had entered onto the 105AD7 study 21 weeks after completion of chemotherapy with cisplatin, doxorubicin and high-dose methotrexate. A routine follow-up chest radiograph performed at week 6 of the vaccine study (and 1 year from original diagnosis) had shown appearance of a single solitary $0.5 \mathrm{~cm}$ nodule, 
Table 2 Clinical responses of patients

\begin{tabular}{|c|c|c|c|c|c|c|c|c|c|}
\hline $\begin{array}{l}\text { Trial } \\
\text { number }\end{array}$ & $\begin{array}{l}\text { Clinical } \\
\text { risk } \\
\text { group }\end{array}$ & $\begin{array}{l}\text { Interval from } \\
\text { last chemo to } \\
\text { vaccination }\end{array}$ & $\begin{array}{l}\text { Immune } \\
\text { response } \\
\text { group }\end{array}$ & $\begin{array}{l}\text { No. of } \\
\text { prior } \\
\text { relapses }\end{array}$ & $\begin{array}{l}\text { Disease at study } \\
\text { entry }\end{array}$ & Subsequent course & $\begin{array}{l}\text { Current } \\
\text { status }\end{array}$ & $\begin{array}{l}\mathbf{F U}^{\mathrm{a}} \\
(\mathbf{m})\end{array}$ & $\begin{array}{l}\text { Date of } \\
\text { last FU } \\
\text { info }\end{array}$ \\
\hline 3 & । & 24 & । & 0 & NMD & Never relapsed & ADF & 45 & 09/04/02 \\
\hline 21 & | & 16 & I & 2 & NMD & Never relapsed & ADF & 34 & | |/| |/2000 \\
\hline 26 & I & 21 & 1 & 0 & NMD & Never relapsed & ADF & 28 & $27 / \mid 2 / 2001$ \\
\hline 28 & 2 & 25 & I & 0 & NMD & Never relapsed & ADF & 24 & $03 / 02 / 2003$ \\
\hline 6 & I & 14 & 1 & 2 & NMD & Nodal and bone relapse & DOD & 18 & $|8 / 10 / 200|$ \\
\hline 12 & i & 12 & i & 0 & NMD & Two further relapses & DOD & 26 & $17 / 10 / 2000$ \\
\hline 22 & I & 22 & 1 & 2 & $\begin{array}{l}\text { Equivocal PD in lungs } \\
\text { on } C T\end{array}$ & PD lungs & DOD & 18 & $04 / 12 / 2002$ \\
\hline 24 & । & 12 & 1 & 3 & Macroscopic residue & Local recurrence and lung mets & DOD & 4 & $25 / 11 / 2002$ \\
\hline 9 & I & 22 & $\|$ & 0 & $\begin{array}{l}\text { Residual inoperable } \\
\text { primary \& metastatic } \\
\text { disease }\end{array}$ & No further progressions & ADF & 49 & $06 / 10 / 2003$ \\
\hline 17 & । & 22 & $\|$ & I & NMD & No further relapse & ADF & 33 & $26 / 06 / 2003$ \\
\hline 23 & 2 & 25 & $\|$ & 0 & NMD & Never relapsed & ADF & 31 & $20 / 07 / 2001$ \\
\hline 20 & । & 21 & $\|$ & 2 & NMD & PD lungs & AWD & 34 & $17 / 10 / 2002$ \\
\hline 7 & । & 20 & $\|$ & । & Local recurrence & Local PD & DOD & 6 & $27 / 01 / 2003$ \\
\hline 16 & 2 & 14 & $\|$ & 0 & NMD & Two further relapses (lung and abdo) & DOD & 19 & $20 / 11 / 2002$ \\
\hline 31 & । & 12 & $\|$ & 2 & NMD & One further relapse & DOD & 8 & $02 /|0 / 200|$ \\
\hline । & 2 & 21 & III & 0 & NMD & pulm mets during vaccination & ADF & 61 & $26 / 10 / 2003$ \\
\hline 5 & । & 27 & III & । & NMD & No further relapse & ADF & 45 & 02/01/03 \\
\hline 10 & । & 25 & III & । & NMD & Two skin relapses in thoracotomy scar & ADF & 32 & $17 / 02 / 2000$ \\
\hline | | & 2 & 8 & III & 0 & NMD & Never relapsed & ADF & 44 & $01 / 01 / 2003$ \\
\hline 29 & 2 & 18 & III & 0 & NMD & Never relapsed & ADF & 26 & $|2 / 09 / 200|$ \\
\hline 2 & I & 19 & III & 3 & NMD & Relapsed, Feb 2002 & AWD & 50 & $20 / 06 / 2002$ \\
\hline 4 & I & 11 & III & 2 & NMD & Further pulm relapse & DOD & 10 & $26 / 06 / 2003$ \\
\hline 8 & । & || & III & 0 & NMD & Three further relapses & DOD & 25 & |3/| |/2002 \\
\hline 13 & । & 9 & III & । & NMD & One further relapse & DOD & 36 & $24 / 05 / 2001$ \\
\hline 14 & । & 7 & III & I & NMD & Further pulm relapse & DOD & 17 & $13 / 02 / 2000$ \\
\hline 15 & । & 22 & III & 4 & PD & Continued PD & DOD & 2 & $22 / 06 / 2001$ \\
\hline 18 & I & 9 & III & 1 & NMD & $\begin{array}{l}\text { Local recurrence, then mediastinal } \\
\text { recurrence, further local recurrence }\end{array}$ & DOD & 16 & 07/07/200 I \\
\hline 27 & I & 23 & III & 2 & NMD & Pulmonary relapse & DOD & 7 & $04 / 02 / 2003$ \\
\hline
\end{tabular}

$\mathrm{NMD}=$ no measurable disease; $\mathrm{PD}=$ progressive disease. ${ }^{\mathrm{a}} \mathrm{FU}$ is months from study entry.

suspicious for metastasis. She completed the fourth dose of vaccine in the interval prior to excision of this nodule for diagnostic purposes, during which time the nodule decreased slightly on chest $\mathrm{X}$-ray. At surgery, both this and a second, $2 \mathrm{~mm}$ nodule found in the other lung were excised completely and found to contain viable osteosarcoma. Despite the early relapse, the patient declined second line chemotherapy but was granted permission to continue a prolonged course of vaccination at 2 monthly intervals over a 2 -year period. Immune response data (Figure 3B) showed a decrease in a pre-existing endogenous response, which was boosted after the third dose of vaccine. It is of note that at this timepoint the patients pulmonary metastasis stabilised. On continued vaccination, her in vitro immune response remained measurable except for a single sample at week 63 . The patient remains free of recurrence 5.2 years from study entry and 3.2 years from last vaccination.

\section{DISCUSSION}

105AD7 is a human anti-idiotypic antibody that binds to the monoclonal antibody $791 \mathrm{~T} / 36$ and mimics the complement regulatory protein CD55. It has previously been shown to induce antitumour inflammatory responses that are associated with tumour cell apoptosis in colorectal cancer patients. As 791T/36 has been shown to stain osteosarcoma tumours and when radiolabelled has been used successfully in diagnostic imaging of these tumours. Therefore, osteosarcoma patients were potential candidates for 105AD7 vaccination. Colorectal cancer patients with minimal residual disease were shown to have better immune responses to 105AD7 than either patients with recurrent disease or patients with a large tumour burden (Durrant et al, 2000a; Maxwell-Armstrong et al, 2001). This may have been due to the immunosuppressive effect of a large tumour burden. The objectives of this study were therefore to assess the immune response to $105 \mathrm{AD} 7$ of children and young adults $(<30$ years of age) when commenced within 1-6 months of finishing systemic chemotherapy. Its main end points were toxicity and immune response, with evaluation of any clinical responses as a secondary end point.

There was no clinically significant toxicity associated with the vaccination even in the two patients who were given compassionate permission to continue being immunised for a 2 -year period. This is in accord with the lack of significant toxicity seen in the colorectal cancer studies in older patients. 105AD7 combined i.d. and i.m. vaccine has now been administered safely to over 200 cancer patients across a wide age range.

The majority of patients (71\%) showed an in vitro $\mathrm{T}$-cell proliferation response to 105AD7 but not to the control human IgG. However, three immunisations were required to induce peak proliferative responses in the majority of patients. This is in contrast to the chemonaive colorectal cancer patients, who showed peak proliferation following their initial vaccination with 105AD7. Previous studies have shown that patients with an HLA-DR 1, 3 or 7 phenotype responded to 105AD7 vaccination. This observation was confirmed in this study with $80 \%$ of patients with these haplotypes showing a proliferation response to 105AD7. However, patients with a DR 13,15 or 17 phenotype also responded suggesting that these haplotypes may also be able to present the class II peptide. This is not uncommon as many class II haplotypes 

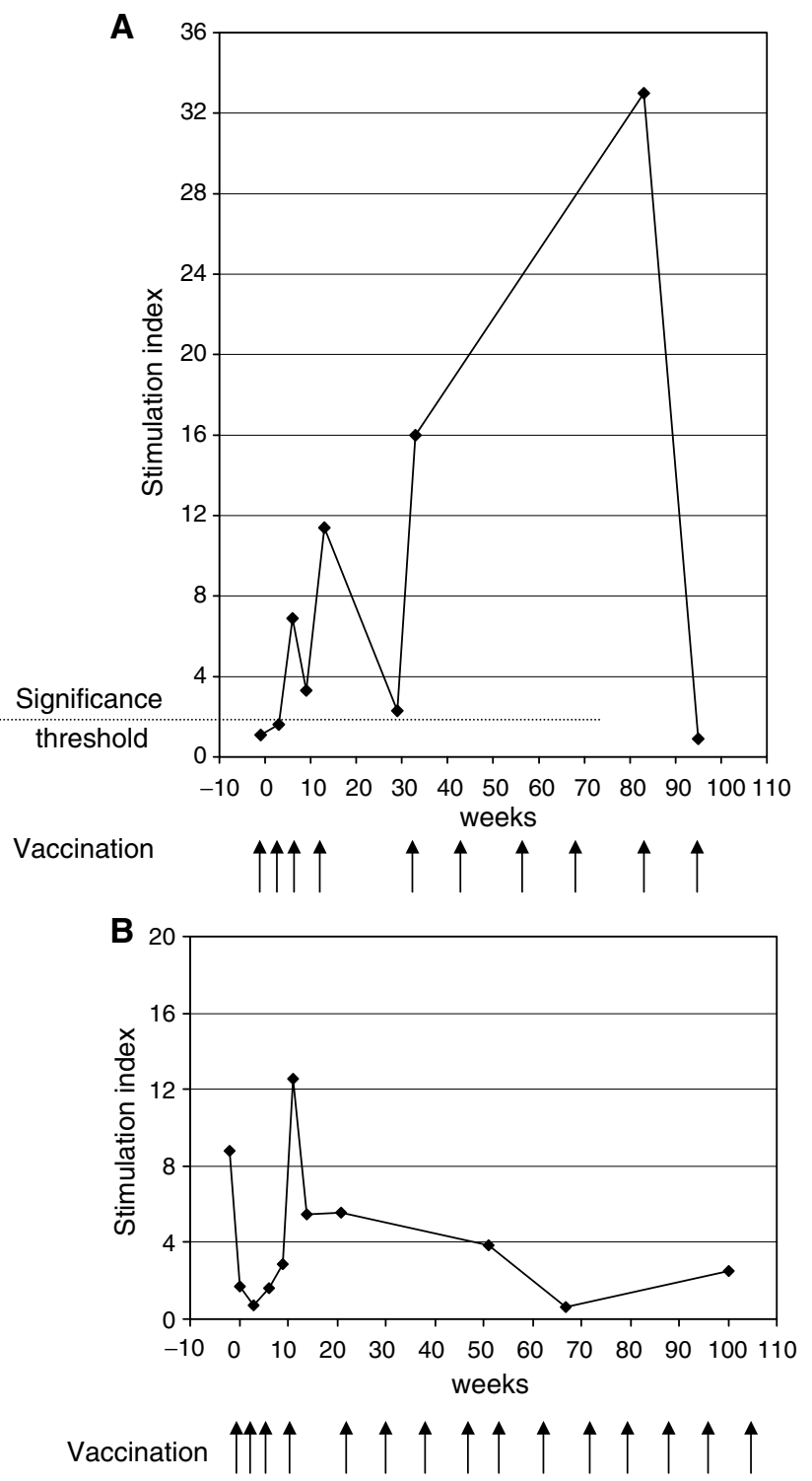

Figure 3 (A) Proliferation response of patient CRC09 following immunisation with I05AD7. (B) Proliferation response of patient CRC0 I following immunisation with I05AD7. T-cell proliferation was assessed by ${ }^{3} \mathrm{H}$-labelled thymidine incorporation following 5-day stimulation with either 105AD7 or control human IgG:

$$
\mathrm{SI}=\frac{\mathrm{cpm} \mathrm{105AD7}}{\mathrm{cpm} \mathrm{hIgG}}
$$

An $\mathrm{SI}$ of greater than 2 is considered significant. Arrows denote injection with 105AD7 by intradermal (10 $\mu \mathrm{g})$ and intramuscular (100 $\mu \mathrm{g}$ on alum) injection.

have similar anchor residue requirements and show promiscuous binding of class II peptides (Chicz et al, 1993).

Lymphocytes responding to 105AD7 also secreted $\gamma$ IFN suggesting that 105AD7 is inducing a Th1 immune response. This is in agreement with the low levels of anti-CD55 antibodies

\section{REFERENCES}

A'Hern RP (2001) Sample size tables for exact single-stage phase II designs. Stat Med 20: 859-866

Altman DG, Machin D, Bryant TN, Gardner MJ (2000) Statistics with Confidence. BMJ Books, BMJ Publishing Group: London induced by this vaccine, suggesting that low-dose immunisation with 105AD7 predominantly induces a cellular Th1 rather than a Th2 response. This is in agreement with our animal studies that showed low doses of 105AD7 induced DTH responses whereas higher doses in combination with complete Freund's adjuvant induced anti-CD55 antibody responses (Austin et al, 1991).

Several patients had an apparent pre-existing proliferation response to $105 \mathrm{AD} 7$ prior to vaccination. In a recent randomised study in colorectal cancer, many of the unimmunised control patients showed an in vitro response to 105AD7 (unpublished results). An alternative suggestion is that disease regression associated with intensive chemotherapy induces an immune response to CD55 that can be detected in vitro with 105AD7. In this context six out of eight patients in group III also had an antibody response to CD55 prior to vaccination. High levels of CD55 released from dying osteosarcoma tumours presented in the context of inflammation may overcome immune ignorance or tolerance associated with this self-antigen. Further studies using antigen-specific ELISPOT assays will determine the frequency and specificity of these T-cell responses.

This trial was not designed to measure significant clinical benefit and only five patients with measurable disease were enrolled. However, disease status was followed in all patients preand postvaccination. Two patients showed evidence of clinical responses. One patient, who entered the study without measurable disease, had early lung metastasis, occurring within 1 year of original diagnosis, which was suspected during immunisation. She continued the vaccine on a compassionate basis for a total of 2 years without any other therapy. This patient remains disease free 4.7 years from time of metastasis. The second patient had chemorefractory primary disease that stabilised on 105AD7 immunisation. Immunisation was continued for a further 2 years and their disease has remained stable for a further 2 years since completion of vaccinations.

A theoretical concern in the design of this study was that the intensive chemotherapy used to treat osteosarcoma might prevent the mounting of a significant immune response when vaccination is commenced within a time frame of 6 months following end of chemotherapy, when patients are still considered immunosuppressed. This study demonstrates that this did not prevent this young group of patients responding to vaccination with $105 \mathrm{AD} 7$.

In conclusion, this study showed that $71 \%$ of young osteosarcoma patients were capable of mounting an immune response to an anti-idiotypic vaccine approach with $105 \mathrm{AD} 7$, commenced within a short time frame (6 months) of completion of intensive chemotherapy. There was no clinically significant toxicity associated with the vaccine. These results suggest that future trials of immunotherapy in osteosarcoma could include a vaccination strategy implemented early, when patients destined to relapse still have minimal disease burden. A randomised phase III study, with progression-free survival as an end point, is necessary to determine if this approach confers any survival benefit in osteosarcoma.

\section{ACKNOWLEDGEMENTS}

CRUK, all patients, research nurses and clinicians at recruiting centres. Magdalen Park Bowling club whose donation paid for transport of specimens. 
Austin EB, Robins RA, Baldwin RW, Durrant LG (1991) Induction of delayed-hypersensitivity to human tumour-cells with a human monoclonal antiidiotypic antibody. J Natl Cancer Inst 83: 1245-1248

Austin EB, Robins RA, Durrant LG, Price MR, Baldwin RW (1989) Human monoclonal anti-idiotypic antibody to the tumour associated antibody 791T/36. Immunology 67: 525-530

Chicz RM, Urban RG, Gorga JC, Vignali DAA, Lane WS, Strominger JL (1993) Specificity and promiscuity among naturally processed peptides bound to HLA-DR alleles. J Exp Med 178: $27-47$

Denton GWL, Durrant LG, Hardcastle JD, Austin EB, Sewell HF, Robins RA (1994) Clinical outcome of colorectal-cancer patients treated with human monoclonal antiidiotypic antibody. Int J Cancer 57: 10-14

Durrant LG, Armstrong CM, Buckley D, Amin S, Robins RA, Carmichael JC, Scholefield JS (2000a) A neoadjuvant clinical trial in colorectal cancer patients of the human anti-idiotypic antibody 105AD7, which mimics CD55. Clin Cancer Res 62: $422-430$

Durrant LG, Spendlove I, Buckley DJ, Robins RA (2000b) 105AD7 cancer vaccine stimulates anti-tumour helper and cytotoxic $T$ cell responses in HLA-A1,3,24 and HLA-DR 1,3,7 colorectal cancer patients. Int J Cancer 85: $87-92$

Farrands PA, Perkins A, Sully L, Hopkins JS, Pimm MV, Baldwin RW, Hardcastle JD (1983) Localisation of human osteosarcomas by antitumour monoclonal antibody. J Bone Jt Surg 65: 638-640

Fleming TR (1982) One-sample multiple testing procedure for Phase II clinical trials. Biometrics 38: 143-151

Fuchs N, Bielack SS, Epler D, Bieling P, Delling G, Korholz D, Graf N, Heise U, Jurgens H, Kotz R, Salzer-Kuntschik M, Weinel P, Werner M, Winkler K (1998) Long-term results of the co-operative German-Austrian-Swiss osteosarcoma study group's protocol COSS-86 of intensive multidrug chemotherapy and surgery for osteosarcoma of the limbs. Ann Oncol 9: $893-899$
Maxwell-Armstrong CA, Durrant LG, Buckley TJD, Scholefield JH, Robins RA, Fielding K, Monson JRT, Guillou P, Calvert H, Carmichael J, Hardcastle JD (2001) Randomized double-blind phase II survival study comparing immunization with the anti-idiotypic monoclonal antibody 105AD7 against placebo in advanced colorectal cancer. Br J Cancer 84: $1443-1446$

Meyers PA, Schwartz CL, Bernstein M, Betcher D, Conrad E, Ferguson W, Gebhardt M, Goodman M, Goorin A, Grier HE, Harris M, Healey J, Huvos A, Kleinerman E, Krailo M, Link M, Montebello J, Nieder M, Sato J, Siegal G, Weiner M, Wells R, Wold L, Womer R, Children's Oncology Group (2001) Addition of ifosfamide and muramyl tripeptide to cisplatin, doxorubicin and high-dose methotrexate improves event-free survival (EFS) in localized osteosarcoma (OS). Proceedings of the American Society for Clinical Oncology, Biometrics 1463a

Price MR, Edwards S, Jacobs E, Baldwin RW (1987) Mapping of monoclonal antibody-defined epitope associated with carcinoembryonic antigen, CEA. Cancer Immunol Immunother 25: 10-15

Schwann A, Robins RA, Maxwell-Armstrong CA, Scholefield JH, Durrant LG (2000) Vaccine-induced apoptosis: a novel clinical trial end point? Cancer Res 60: $3132-3136$

Souhami RL, Craft AW, Van der Eijken JW, Nooij M, Spooner D, Bramwell VH, Wierzbicki R, Malcolm AJ, Kirkpatrick A, Uscinska BM, Van Glabbeke M, Machin D (1997) Randomized trial of two regimens of chemotherapy in operable osteosarcoma: a study of the European Osteosarcoma Intergroup. Lancet 9: 911-917

Spendlove I, Li L, Carmichael J, Durrant LG (1999) Decay accelerating factor (CD55): a target for cancer vaccines? Cancer Res 59: 2282-2286

Spendlove I, Li L, Potter V, Christiansen D, Loveland B, Durrant LG (2000) A therapeutic human anti-idiotypic antibody (105AD7) mimics CD55 (decay accelerating factor) in three distinct regions. Eur J Immunol 30: $2944-2953$ 